\title{
Recurrent Mucinous Ovarian Neoplasms: Rationale and Results of a New Treatment Plan in Four Patients
}

\author{
Sugarbaker $\mathrm{PH}^{* 1}$ and Dainty $\mathrm{LA}^{2}$ \\ ${ }^{1}$ Program in Peritoneal Surface Oncology, MedStar Washington Cancer Institute and MedStar Washington \\ Hospital Center, Washington DC, USA \\ ${ }^{2}$ Department of Gynecologic Oncology, MedStar Washington Hospital Center, Washington DC, USA
}

*Corresponding author: Sugarbaker PH, MD, FACS, FRCS, 106 Irving St, NW, Suite 3900, Washington, DC 20010, USA, Fax: +1-202-877-7287, Tel: +1-202-877-3908, E-mail: Paul.Sugarbaker@medstar.net

Citation: Sugarbaker PH, Dainty LA (2019) Recurrent Mucinous Ovarian Neoplasms: Rationale and Results of a New Treatment Plan in Four Patients. J Case Rep Stud 7(2): 208. doi: 10.15744/2348-9820.7.208

Received Date: February 28, 2019 Accepted Date: April 25, 2019 Published Date: April 27, 2019

\begin{abstract}
Objective: Recurrence of a mucinous ovarian neoplasm after resection is unusual but this does happen. If possible, a repeat surgical removal of recurrent disease is the treatment of choice. The results of a repeat intervention are poor. A review of our results of treatment of recurrent mucinous ovarian neoplasms was performed.

Methods: Our database of peritoneal surface malignancy patients was reviewed and those with mucinous ovarian neoplasms identified. The clinical features, surgical procedures and chemotherapy treatments were reviewed. All follow-ups were updated.

Results: Four patients with recurrent mucinous ovarian neoplasms were identified. These were primary in the ovary and not metastatic from a gastrointestinal primary site. Three were borderline and one was an invasive neoplasm. Resection using peritonectomy to remove all visible disease maximized clearance and containment in all four patients. Also, extensive intraperitoneal lavage or perioperative intraperitoneal chemotherapy was used in an attempt eliminate microscopic residual disease. Three patients have remained free of disease for 23, 16, and 13 years. The fourth patient is now free of disease but less than 6 months postoperative.

Conclusion: Management of recurrent mucinous ovarian neoplasms with resections that utilize peritonectomy and strategies to eliminate microscopic residual disease was associated with long-term disease-free survival. Benefits seen in these patients may have implications for management of all recurrent mucinous ovarian neoplasms.
\end{abstract}

Keywords: Cytoreductive Surgery; HIPEC; EPIC; Extensive Intraperitoneal Lavage; Peritonectomy; Borderline Malignancy

\section{Introduction}

According to Seidman et al. borderline mucinous tumors comprise $1.0 \%$ of benign ovarian epithelial neoplasms. Invasive ovarian mucinous neoplasms comprise 3\% of invasive ovarian carcinomas [1]. The low grade tumors often occur in younger women and are usually definitively treated by laparoscopic or open resection. Although unusual, recurrence does happen; this is estimated at $8 \%$ of cases [2]. When recurrence is diagnosed a reoperative surgical procedure may be indicated. Progression of a recurrent borderline mucinous neoplasm may be slow. Recurrence of an invasive mucinous ovarian carcinoma may be more aggressive and has a poor outcome $[3,4]$. Other treatments such as radiation therapy or chemotherapy are regarded as usually ineffective for recurrence of both low grade and high grade neoplasms [5]. Precise recommendations for management of recurrent mucinous ovarian neoplasms other than reoperative surgery have not been published. Long-term results with treatment of recurrent borderline disease are not available. This manuscript presents the experience with 4 patients who had recurrent mucinous ovarian neoplasms. Long-term survival in the absence of recurrence is reported.

\section{Materials and methods}

A retrospective review of a prospective database of 1721 patients treated for peritoneal metastases was available. Seventy of these patients were treated for ovarian malignancy and 64 of these were for recurrent ovarian cancer. Only 4 of these had a recurrent mucinous ovarian neoplasm. Demographic information and clinical data were obtained from the patient database. Surgical procedures performed and pathology reports were available for review. Continuous follow-up information was available on all 4 patients. 
Cytoreductive surgery with peritonectomy was performed by laparotomy through a long midline abdominal incision [6]. Peritonectomy provided a pre-peritoneal approach to disease recurrence in order to minimize the possibility for small volume residual disease. Perioperative intraperitoneal chemotherapy was used if peritoneal metastases were at several sites [7]. If peritoneal recurrence was limited to a single site extensive intraperitoneal lavage was used [8]. Chemotherapy used for intraperitoneal administration was doxorubicin and cisplatin in two patients and melphalan in a third patient [9].

Data on these 4 patients was prospectively recorded and then retrospectively reviewed at an academic institution. All information was from a single institution and the cases were consecutive. This research work has been reported in line with the PROCESS criteria [10]. This study was registered as a case series on the www.researchregistry.com website with UIN 4409.

\section{Patient presentation}

Patient 1: A 30 year old woman developed an expanding mass while pregnant. In February 1994, at 26 weeks gestation, a transverse lower abdominal incision was made and the abdomen opened vertically. The $12 \mathrm{~cm}$ left ovarian mass was fixed in the pelvis. As the left ovary was being removed it ruptured and grossly contaminated the abdomen and pelvis. Pathology showed a mucinous borderline cystadenoma of the ovary with focal microinvasion. The patient had a normal labor and delivery.

In January 1995, the patient was electively taken back to the operating room for a second-look procedure. A $2 \mathrm{~cm}$ mass was removed from the cul-de-sac. A margin of excision was obtained using pelvic peritonectomy with sparing of the rectosigmoid colon. By pathologic examination this showed a metastatic mucinous cystic neoplasm of low malignant potential. Mucinous adenocarcinoma could not be ruled out. Drains and catheters were placed for early postoperative intraperitoneal chemotherapy (EPIC) with cisplatin and doxorubicin [7]. The surgery and perioperative intraperitoneal chemotherapy were tolerated well and the patient recovered without incident.

Early in 1997, the patient complained of right lower quadrant discomfort. By CT there was an $8 \mathrm{~cm}$ mass in the position of the right ovary. In March of 1997 the patient was again explored through a midline abdominal incision. The right ovarian mass was adherent to the surrounding structures. A greater omentectomy, pelvic peritonectomy and difficult right ureterolysis were performed to resect the mass en bloc. A hysterectomy was performed. Extensive intraperitoneal lavage was used to assist in the control of microscopic residual disease [8]. Because it was possible to perform an resection, perioperative intraperitoneal chemotherapy was not used.

Again, pathology showed a borderline mucinous tumor of low malignant potential of Mullerian type. There were foci of superficial invasion.

The patient recovered well following her third surgery and remains no evidence of disease with a normal quality of life 23 years later.

Patient 2: A 74 year old woman in March of 1998 had a hysterectomy and right oophorectomy for benign disease. Then, in April of 2001, she developed a swelling of her left lower extremity, which was caused by a deep vein thrombosis. She had a vena cava filter placed. In the same month she had an abdominal exploration and removal of the left ovary. Pathology showed mucinous carcinoma.

In September 2001, the mass in the left pelvis recurred. By CT the lungs and upper abdomen, as well as the liver, were normal. There was a $14 \mathrm{~cm}$ mass that filled the pelvis and caused obstruction of the left ureter. There was some function of this kidney by CT, but renogram showed that the function was greatly reduced.

In October 2001 the patient underwent a six-hour cytoreductive surgery. En bloc resection of this large solitary mass required greater omentectomy, pelvic peritonectomy, bilateral ureterolysis, excision of apex of vagina and low colorectal anastomosis. Thorough washing of the abdominopelvic space with 3 liters of $0.2 \%$ peroxide solution and an extensive intraperitoneal lavage (10 liters) with normal saline was performed [8]. The intraabdominal and pelvic irrigation was performed prior to low colorectal anastomosis and abdominal closure. A diverting ileostomy was avoided [11].

Pathology showed a mucinous adenocarcinoma of the left ovary invading the wall of the colon. The vaginal apex was not invaded and six peri-tumoral lymph nodes were negative. The patient's postoperative course was uneventful. She remains free of disease with a normal quality of life 16 years later.

Patient 3: This 34 year old woman, as a very young child, had serous internal injuries to her abdomen after falling down stairs. This resulted in multiple adhesions. Then in early 2002, she noted a swollen abdomen. A gynecologist performed debulking surgery through a Pfannenstiel incision in April 2002. Pathology showed malignant cells compatible with adenocarcinoma. Cyst fluid also showed adenocarcinoma. Shortly thereafter, this tumor started to progress. In September of 2004 she was requiring repeated paracentesis. The abdomen was massively distended with bilateral ureteral obstruction and rectosigmoid colon obstruction from external compression. 
In September 2004, the patient was taken to the operating room for cytoreductive surgery. She underwent 8 hours of surgery which included greater omentectomy, pelvic peritonectomy, hysterectomy and bilateral salpingo-oophorectomy, and rectosigmoid colon resection with low anastomosis. A diverting ostomy with the low colorectal anastomosis was avoided without complications [11]. En bloc resection was not possible and there was extensive tumor dissemination at the time of cytoreductive surgery. Consequently, hyperthermic intraperitoneal chemotherapy (HIPEC) with $24 \mathrm{mg}$ of doxorubicin and $80 \mathrm{mg}$ of cisplatin was completed prior to colorectal anastomosis and abdominal closure. Immunocytochemistry was positive for CEA and B72.3 and negative for cytokeratin $5 / 6$ and calretinin. The diagnosis was massive mucinous ovarian neoplasm.

The patient recovered without incident from cytoreductive surgery plus HIPEC. She has no evidence of disease and a normal quality of life 13 years later.

Patient 4: This 65 year old woman was diagnosed with a right-sided adnexal mass in 1985. A total abdominal hysterectomy and bilateral oophorectomy was performed without incident. In July 2011, a pelvic mass was diagnosed and a resection was performed. The pathology showed a benign mucinous ovarian neoplasm. By CT a pelvic mass was imaged and in July 2017 the patient returned to the operating room for re-excision. The surgery was complicated by multiple enterotomies, deep vein thrombosis, and 8 weeks of hospitalization plus rehabilitation.

Repeat CT in April 2018 showed a bilateral right pelvic cystic mass $12 \mathrm{~cm}$ in greatest diameter and partial obstruction of the right ureter and distorting the bladder and midrectum. Moderate pain was reported. In May 2018, the patient was taken back to the operating room. She had a pelvic peritonectomy, right ureterolysis and resection of a $10 \mathrm{~cm}$ cystic tumor. No bowel resection was necessary. The cystic tumor mass could not be removed intact and gross tumor contamination was caused by a piecemeal resection. Prior to abdominal closure HIPEC with melphalan was used as an abdominal-pelvic lavage [12].

Postoperatively the patient suffered from repeat aspiration requiring a 26-day hospitalization and 3 weeks in a rehabilitation facility. The patient has recovered and is currently fully ambulatory at one year postoperatively.

Review of the pathology of all three resections shows low grade mucinous neoplasm consistent with a borderline mucinous ovarian neoplasm.

(Table 1) presents clinical features of these 4 patients. Our patients with recurrent mucinous ovarian neoplasm did not recur again despite extensive borderline disease in 3 patients and a grossly invasive mucinous neoplasm into the rectosigmoid colon in the third patient. The surgical approach to these patients with recurrent disease was an open laparotomy with wide exposure. Peritonectomy provided a pre-peritoneal approach to disease fixed by tumor or by fibrous adhesions within the pelvis. In 2 of the 4 patients in order to contain and clearly resect the tumor mass completely, a rectosigmoid colon resection was necessary.

\begin{tabular}{|c|c|c|c|c|}
\hline & \multicolumn{4}{|c|}{ Patient } \\
\hline Age at diagnosis & $\mathbf{1}$ & $\mathbf{2}$ & $\mathbf{3}$ & $\mathbf{4}$ \\
\hline $\begin{array}{c}\text { Interval first to definitive } \\
\text { surgery (months) }\end{array}$ & 11 & 74 & 34 & 85 \\
\hline Invasion & Superficial & Gross invasion & Superficial & Superficial \\
\hline Bilateral & Yes & No & Yes & No \\
\hline $\begin{array}{c}\text { Perioperative Chemotherapy/ } \\
\text { lavage }\end{array}$ & EPIC & EIPL & HIPEC & HIPEC \\
\hline $\begin{array}{c}\text { Rectosigmoid colon resection } \\
\text { Time to first recurrence } \\
\text { (months) }\end{array}$ & None & Yes & Yes & No \\
\hline $\begin{array}{c}\text { Survival (years) } \\
\text { (migr| }\end{array}$ & 22 & 6 & 27 & 72 \\
\hline
\end{tabular}

EPIC = Early Postoperative Intraperitoneal Chemotherapy; EIPL = Extensive Intraperitoneal Lavage; HIPEC = Hyperthermic Intraperitoneal Chemotherapy; ${ }^{*}=$ Patient Recently Placed into Follow-Up Table 1: Clinical features of 4 patients with recurrent mucinous ovarian neoplasm

As shown in Table 1, all 4 of our patients had perioperative chemotherapy or extensive lavage to eradicate microscopic residual disease at the resection site and at narrow margins of excision. In all patients en bloc resection was attempted but was not always possible. These treatments were administered after the cytoreductive surgery but before intestinal reconstruction or abdominal closure. Patient 1 received EPIC at her first cytoreduction and extensive lavage at a second cytoreduction. Patient 2 had extensive intraperitoneal lavage (EIPL). Patient 3 and 4 had hyperthermic intraperitoneal chemotherapy (HIPEC).

\section{Discussion}

The results of surgical resection of a primary borderline (low grade) mucinous ovarian neoplasm are usually excellent. Resection in the absence of tumor disruption and spillage is the proper treatment. Adjuvant radiation and chemotherapy is unlikely to 
add a therapeutic advantage [5]. In the small proportion of patients who recur, the optimal treatment strategies have not been defined and the results of simple reoperative surgery have not been published. With recurrence of a borderline mucinous ovarian neoplasm, a repeat surgical procedure is indicated but the surgical approach has not been described. Also, perioperative treatments to minimize the likelihood of microscopic residual disease that may cause another episode of recurrence have not been reported.

The surgical approach that was used on our 3 patients with recurrent borderline mucinous ovarian neoplasm showed long-term favorable results in 2 and the third patient is free of disease in follow-up of less than 6 months. Cytoreductive surgery with peritonectomy and visceral resections were used as needed to avoid positive margins of resection and minimize residual mucinous tumor cells. The second nuance that was utilized for these recurrent borderline ovarian mucinous neoplasms was a perioperative intraperitoneal chemotherapy. This was to minimize the possibility of residual tumor cells that may remain after the best efforts of tumor resection. The bed of the tumor resection and narrow margins of resection are at the greatest risk for subsequent disease progression from microscopic tumor deposits. The local-regional strategy in one patient was EPIC and extensive intraperitoneal lavage and in the other 2 was HIPEC. The optimal perioperative treatment for eradication of microscopic disease that is maximally effective and maximally safe has not as yet been determined.

All 3 of our patients with borderline mucinous ovarian neoplasm had a large extent of recurrent minimally invasive mucinous ovarian neoplasm. The disease was recurrent after a prior maximal surgical effort but in the absence of complete cytoreduction and perioperative intraperitoneal chemotherapy. The outcome of such patients has not been well defined but repeated efforts of debulking surgery have been expected in this clinical situation. With complete cytoreduction to no visible disease combined with perioperative intraperitoneal chemotherapy, these patients have not required further interventions at 22 and 13 years postrecurrence.

In one of these recurrent mucinous ovarian neoplasms the disease was high grade and invasive into the rectosigmoid colon. Resection en bloc with pelvic peritonectomy and rectosigmoid colon resection was possible. Because of the absence of peritoneal metastases the cancer resection was augmented by EIPL [8]. In this single patient this combined treatment resulted in a long-term benefit without adverse events.

The literature supports a poor prognosis of patients who develop a recurrence of a mucinous ovarian neoplasm. Cheng and coworkers identified 21 patients with a secondary cytoreduction for recurrent mucinous epithelial ovarian cancer [3]. Only 7 of these 21 patients were optimally cytoreduced to residual disease less than or equal to one $\mathrm{cm}$. The median survival time from diagnosis of recurrent disease was 10 months. There was no significant difference in median survival between patients with optimal or suboptimal secondary cytoreduction. Kajiyama and coworkers reported on an extremely poor post-recurrence outcome of 44 patients with recurrent ovarian mucinous neoplasms [4]. The five-year survival was only $6.9 \%$ and this was significantly poorer than survival of patients with recurrent serous ovarian cancer $(\mathrm{p}=0.0006)$. These authors conclude that the clinical outcome after recurrence of patients with recurrent mucinous ovarian cancer is extremely poor. Lederman and coworkers published a consensus review of mucinous ovarian carcinoma. They suggested that the poor prognosis with relapse was related to the significantly poorer response to platinum-based chemotherapy of the mucinous histology [5].

In our 4 patients a cytoreductive approach to tumor resection combined with perioperative intraperitoneal lavage using chemotherapy or extensive irrigation was used to treat recurrent mucinous ovarian neoplasms. However, there may be some clinical situations that may profit from a perioperative treatment for microscopic residual disease in the first resection of an ovarian mucinous neoplasm. For example, our patient 1, the large tumor ruptured as it was being resected. There was irrigation but evidently not enough to prevent recurrent disease within the cul-de-sac. If the tumor mass has caused spillage of tumor cells either prior to or at the time of resection, a perioperative intraperitoneal lavage may improve the long-term disease-free survival. It is not surprising that Ben-Ami and coworkers reported that intraoperative cyst rupture and cystectomy instead of adnexectomy are two risk factors for recurrence of borderline mucinous ovarian neoplasms [2].

In the past, cytoreductive surgery with perioperative treatments to combat residual microscopic disease has not been used for the management of recurrent ovarian neoplasms. Cytoreductive surgery consists of a complete resection of all visible disease from the abdomen and pelvis and requires peritonectomy and resection of affected viscera [6,13]. Subsequently, lavage or intraperitoneal chemotherapy is used to eliminate tumor cells that remain viable. Currently, diseases considered for this combined treatment are pseudomyxoma peritonei [14], peritoneal mesothelioma [15], and peritoneal metastases of appendiceal [16], colorectal [17], and ovarian [18,19] origin. Currently, neither borderline nor aggressive ovarian mucinous neoplasms are considered an indication for these combined treatments. However, interest in unusual application of cytoreductive surgery and perioperative chemotherapy has been published [20]. Goere and coworkers reported on 850 procedures for unusual cases performed in 781 patients, in 53 centers worldwide. Nearly two-thirds of the procedures were performed for three indications: rare ovarian carcinoma ( $\mathrm{n}=224)$, sarcoma $(n=189)$ and neuroendocrine tumors $(n=127)$. For the three main indications, 5-year overall survival was significantly greater in patients with peritoneal metastases from rare ovarian carcinoma (57.7\%), than that of patients with peritoneal metastases from neuroendocrine tumors $(39.9 \%)$, and from sarcoma $(29.3 \%)(\mathrm{p}<0.0001)$. These combined treatments appear to be safe and effective in patients with peritoneal metastases from unusual cancer sites of origin, and of special benefit from rare ovarian neoplasms. 
Mucinous neoplasms progressing as peritoneal metastases within the abdomen and pelvis have several characteristics in common. These similarities occur despite different primary sites of the disease. Mucinous peritoneal metastases may arise from the ovary, as in the 4 patients presented here, from the appendix [16], from the colon or rectum [17], from the urachus [21], and from other less common primary sites [22]. Perhaps the characteristic most evident to a surgeon attempting to optimally cytoreduce mucinous peritoneal tumor is the relative sparing of visceral (especially small bowel peritoneal surfaces) as compared to parietal peritoneal surfaces. A large extent of disease may be present in the pelvis, paracolic sulcus, and in sub diaphragmatic spaces while small bowel surfaces remain disease-free [23]. The pattern of peritoneal metastases may have implications for attempts at a visibly complete cytoreduction.

A second characteristic that mucinous tumors with recurrent peritoneal metastases have in common is a wide spectrum of biologic behavior. They may be invasive, of low malignant potential (borderline), or benign (non-invasive). In mucinous peritoneal metastases, the extent of invasive biologic behavior may have profound implications for the success vs. failure of cytoreductive surgery [22].

A third characteristic common to mucinous peritoneal tumors from different primary sites is the lack of response to systemic chemotherapy. Mucinous ovarian cancer responds poorly or not at all to systemic chemotherapy [5]. Appendiceal mucinous neoplasms, low grade or high grade, respond poorly to systemic chemotherapy and are treated with systemic chemotherapy only as no other treatment options exist [24]. Lederman and coworkers, in their consensus statement on mucinous ovarian carcinoma, identify the development of new and better systemic treatments as a highest priority for this disease [5].

Although new systemic treatments are currently not available pharmacologic studies suggest that intraperitoneal drug delivery is expected to be more effective in mucinous as compared to non-mucinous tumors. Pharmacologic studies have shown that intraperitoneal chemotherapy enters into mucinous tumor nodules to a greater extent than into firm peritoneal nodules [25]. Clinical support for this pharmacologic data exists. Bruin and colleagues showed that mucinous appendiceal neoplasms treated with hyperthermic intraperitoneal chemotherapy survived better than appendiceal malignancies that lacked mucinous histology [26]. The marked success in management of a predominantly mucinous neoplasm, pseudomyxoma peritonei, using cytoreductive surgery with perioperative intraperitoneal chemotherapy further supports the hypothesis that intraperitoneal chemotherapy is more effective for mucinous as compared to non-mucinous peritoneal metastases [25]. If this same pharmacologic step up in chemotherapy diffusion into mucinous ovarian tumors exists, intraperitoneal chemotherapy may be as effective for mucinous ovarian tumors as for mucinous appendiceal neoplasms.

Support for the new treatment plan for recurrent mucinous tumors is found in a recent manuscript by Mercier et al. [27]. They corrected retrospective data on 77 mucinous ovarian tumors. The grade of the cancer was not provided in the manuscript. All patients had an attempt at complete cytoreductive surgery and all patients had the HIPEC procedure. A 5-year survival of 69.6\% was reported. These authors conclude, as our results also suggest, that long-term survival with complete cytoreductive surgery plus HIPEC is possible with recurrent mucinous ovarian tumors. Of course, en bloc resection is always a highest priority.

\section{Summary}

Although our report of these patients and the accompanying literature review suggest that a favorable outcome may be possible, no randomized studies on recurrent mucinous ovarian neoplasms exist. All patients with a favorable outcome were approached surgically with an attempt to remove all visible evidence of disease. The goal of resection of tumor was not resection of all tumor nodules to $1 \mathrm{~cm}$ or less in diameter. Rather the goal of surgery was a complete visible clearance of disease. The goal for optimal cytoreduction in these patients as compared to surgical goals in the past was markedly different. A second difference in treatment strategy involved a perioperative irrigation with HIPEC to eradicate microscopic residual cancer cells that may persist after a major dissection that involves much of the abdomen and the entire pelvis. This is the same strategy that has been associated with success with several other mucinous tumor types $[14,16,21,22]$. The success of this new treatment strategy for recurrent mucinous ovarian neoplasms is in marked contrast to the dismal outcome reported in the past [3-5]. Until more data becomes available complete cytoreductive surgery plus a HIPEC procedure may be considered a preferred treatment option in these patients.

\section{Conflict of interest}

The authors have no conflicts of interest to declare.

\section{Author contributions}

Both authors contributed to the conceptualization, formal analysis, investigation, methodology, resources, supervision, validation, writing of original draft and review and editing.

\section{References}

1. Barakat RR, Berchuck A, Markman M, Randall M (2013) Epithelial ovarian cancer. In: Principles and Practice of Gynecologic Oncology (6 $6^{\text {th }}$ Edn) Philadelphia: Wolters Kluwer, USA. 
2. Ben-Ami I, Smorgick, N, Tovbin J, Fuchs N, Halperin R, et al. (2010) Does intraoperative spillage of benign ovarian mucinous cystadenoma increase its recurrence rate? Am J Obstet Gynecol 202: 142.e1-5.

3.Cheng X, Jiang R, Li ZT, Tang J, Cia SM, et al. (2009) The role of secondary cytoreductive surgery for recurrent mucinous epithelial ovarian cancer (mEOC). Eur J Surg Oncol 35: 1105-8.

4. Kajiyama H, Mizuno M, Shibata K, Kawai M, Nagasaka T, et al. (2014) Extremely poor postrecurrence oncological outcome for patients with recurrent mucinous ovarian cancer. Int J Clin Oncol 19: 121-6.

5. Lederman JA, Luvero D, Shafer A, O'Connor D, Mangili G, et al. (2014) Gynecologic Cancer InterGroup (GCIG) consensus review for mucinous ovarian carcinoma. Int J Gynecol Cancer 24: S14-S19.

6. Sugarbaker PH (1995) Peritonectomy procedures. Ann Surg 221: 29-42.

7. van der Speeten K, Stuart OA, Sugarbaker PH (2017) Cancer Chemotherapy for Peritoneal Metastases: Pharmacology and Treatment. In: Cytoreductive Surgery \& Perioperative Chemotherapy for Peritoneal Surface Malignancy. Textbook and Video Atlas ( $2^{\text {nd }}$ Edn) Woodbury, CT: Cine-Med Publishers, USA.

8. Kuramoto M, Shimada S, Ikeshima S, Matsuo A, Yagi Y, et al. (2009) Extensive intraoperative peritoneal lavage as a standard prophylactic strategy for peritoneal recurrence in patients with gastric carcinoma. Ann Surg 250: 242-6.

9. Sugarbaker PH, van der Speeten K (2016) Surgical technology and pharmacology of hyperthermic perioperative chemotherapy. J Gastrointest Oncol 7: 29-44. 10. Agha RA, Fowler AJ, Rammohan S, Barai I, Orgill DP, et al. (2016) Preferred Reporting of Case Series in Surgery; the PROCESS guidelines. Int J Surg 36: 319-23. 11. Bijelic L, Sugarbaker PH, Stuart OA (2012) Hyperthermic intraperitoneal chemotherapy with melphalan: a summary of clinical and pharmacologic data in 34 patients. Gastroenterol Res Pract: 10.1155/2012/827534.

12. Sugarbaker PH (2016) Avoiding a diverting ileostomy in patients requiring complete pelvic peritonectomy. Ann Surg Oncol 23: $1481-5$.

13. Sugarbaker PH, van der Speeten K (2017) An Overview of Peritonectomy, Visceral Resection, and Therapeutic Laparoscopy for Peritoneal Surface Malignancy. In: Cytoreductive Surgery \& Perioperative Chemotherapy for Peritoneal Surface Malignancy. Textbook and Video Atlas (2 ${ }^{\text {nd }}$ Edn) Woodbury, CT: Cine-Med Publishers, USA.

14. Sugarbaker PH (2006) New standard of care for appendiceal epithelial malignancies and pseudomyxoma peritonei syndrome. Lancet Oncol 7: 69-76.

15. Sugarbaker PH, Turaga KK, Alexander HR Jr, Deraco M, Hesdorffer M (2016) Management of malignant peritoneal mesothelioma using cytoreductive surgery and perioperative chemotherapy. J Oncol Pract 12: 928-35.

16. Sugarbaker PH, Chang D (1999) Results of treatment of 385 patients with peritoneal surface spread of appendiceal malignancy. Ann Surg Oncol 6: 727-31.

17. Sugarbaker PH (1999) Successful management of microscopic residual disease in large bowel cancer. Cancer Chemother Pharmacol 43(Suppl): S15-S25.

18. Bakrin N, Bereder JM, Decullier E, Classe JM, Msika S, et al. (2013) Peritoneal carcinomatosis treated with cytoreductive surgery and Hyperthermic Intraperitoneal Chemotherapy (HIPEC) for advanced ovarian carcinoma: a French multicentre retrospective cohort study of 566 patients. Eur J Surg Oncol 39 : $1435-43$.

19. Spiliotis J, Halkia E, Lianos E, Kalantzi N, Grivas A, et al. (2015) Cytoreductive surgery and HIPEC in recurrent epithelial ovarian cancer: A prospective randomized phase III study. Ann Surg Oncol 22: 1570-5.

20. Goéré D, Passot G, Gelli M, Levine EA, Bartlett DL, et al. (2017) Complete cytoreductive surgery plus HIPEC for peritoneal metastases from unusual cancer sites of origin: results from a worldwide analysis issue of the Peritoneal Surface Oncology Group International (PSOGI). Int J Hyperthermia 33: 520-7.

21. Mercier F, Passot G, Villeneuve L, Levine EA, Yonemura Y, et al. (2018) Peritoneal Carcinomatosis of Urachus Origin Treated by Cytoreductive Surgery and Hyperthermic Intraperitoneal Chemotherapy (HIPEC): An International Registry of 36 Patients. Ann Surg Oncol 25: $1094-100$.

22. Sugarbaker PH (2017) Treatment of pseudomyxoma peritonei from non-appendiceal primary sites. Unusual Cases in Peritoneal Surface Malignancies, Switzerland: 23-46.

23. Carmignani CP, Sugarbaker TA, Bromley CM, Sugarbaker PH (2003) Intraperitoneal cancer dissemination: mechanisms of the patterns of spread. Cancer Metastasis Rev 22: 465-72.

24. Blackham AU, Swett K, Eng C, Sirintrapun J, Bergman S, et al. (2014) Perioperative systemic chemotherapy for appendiceal mucinous carcinoma peritonei treated with cytoreductive surgery and hyperthermic intraperitoneal chemotherapy. J Surg Oncol 109: 740-5.

25. Van der Speeten K, Stuart OA, Sugarbaker PH (2009) A pharmacologic analysis of intraoperative intracavitary cancer chemotherapy with doxorubicin. Cancer Chemother Pharmacol 63: 799-805.

26. Bruin SC, Verwaal VJ, Vincent A, van't Veer LJ, van Velthuysen ML (2010) A clinicopathologic analysis of peritoneal metastases of colorectal and appendiceal origin. Ann Surg Oncol 17: 2330- 40.

27. Mercier F, Bakrin N, Bartlett DL, Goere D, Quenet F, et al. (2018) Peritoneal Carcinomatosis of Rare Ovarian Origin Treated by Cytoreductive Surgery and Hyperthermic Intraperitoneal Chemotherapy: A Multi-Institutional Cohort from PSOGI and BIG-RENAPE. Ann Surg Oncol 25: $1668-75$.

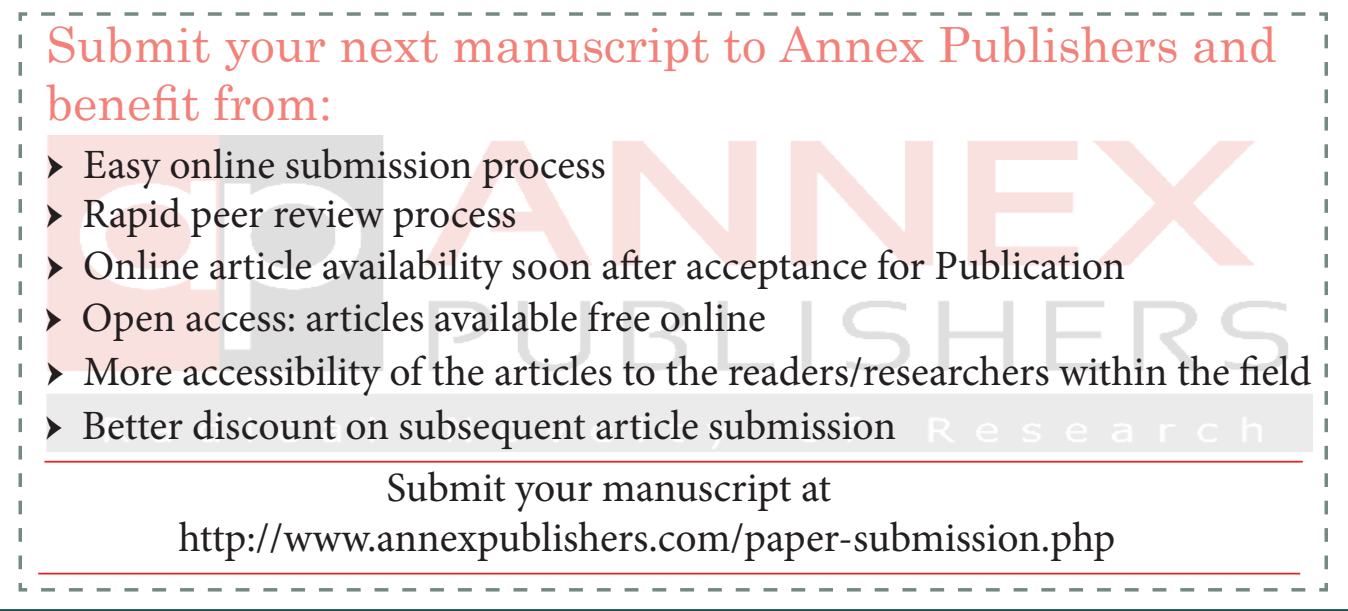

